\section{OPEN ACCESS}

Edited by:

Cheng Zhong

Tianjin University, China

Reviewed by:

Jia Ding,

Tianiin University, China

Jin Huile,

Wenzhou University, China

*Correspondence:

Guoqiang Zou

gq-zou@csu.edu.cn

Specialty section:

This article was submitted to

Electrochemistry,

a section of the journal

Frontiers in Chemistry

Received: 04 December 2019 Accepted: 14 January 2020

Published: 04 February 2020

Citation:

Cai P, Zou K, Deng X, Wang B, Zou G, Hou H and Ji X (2020) Defect Rich Hierarchical Porous Carbon for High

Power Supercapacitors.

Front. Chem. 8:43

doi: $10.3389 /$ fchem.2020.00043

\title{
Defect Rich Hierarchical Porous Carbon for High Power Supercapacitors
}

\author{
Peng Cai ${ }^{1}$, Kangyu Zou ${ }^{1}$, Xinglan Deng ${ }^{1}$, Baowei Wang ${ }^{1}$, Guoqiang Zou ${ }^{1 *}$, \\ Hongshuai Hou ${ }^{1}$ and Xiaobo $\mathrm{Ji}^{1,2}$
}

${ }^{1}$ College of Chemistry and Chemical Engineering, Central South University, Changsha, China, ${ }^{2}$ School of Materials Science and Engineering, Zhengzhou University, Zhengzhou, China

Tuning hierarchical pore structure of carbon materials is an effective way to achieve high energy density under high power density of carbon-based supercapacitors. However, at present, most of methods for regulating pores of carbon materials are too complicated to be achieved. In this work, a durian shell derived porous carbon (DSPC) with abundant porous is prepared through chemical activation as a defect strategy. Hierarchical porous structure can largely enhance the transfer rate of electron/ion. Furthermore, DSPC with multiple porous structure exhibits excellent properties when utilized as electrode materials for electric double layer capacitors (EDLCs), delivering a specific capacitance of $321 \mathrm{~F} \mathrm{~g}^{-1}$ at $0.5 \mathrm{~A} \mathrm{~g}^{-1}$ in aqueous electrolyte. Remarkably, a high energy density of 27.7 $\mathrm{Wh} \mathrm{kg}^{-1}$ is obtained at $675 \mathrm{~W} \mathrm{~kg}^{-1}$ in an organic two-electrode device. And large capacity can be remained even at high charge/discharge rate. Significantly, hierarchical porous structure allows efficient ion diffusion and charge transfer, resulting in a prominent cycling stability. This work is looking forward to providing a promising strategy to prepare hierarchical porous carbon-based materials for supercapacitors with ultrafast electron/ion transport.

Keywords: high power density, hierarchical porous carbon, supercapacitors, biomass carbon, EDLC

\section{INTRODUCTION}

Supercapacitors (SCs) have been triggered intensive attention in energy storage due to their fast reaction dynamics, ultrahigh power density, and excellent cycling stability (Li et al., 2011; Xie et al., 2012; Hao et al., 2013; Wang and Xia, 2013; Béguin et al., 2014). Among various kinds of electrode materials for SCs, carbon materials are most promising because of their large availability, high conductivity, and cycling stability. Preparing carbon materials economically and efficiently, increasing the energy density, and enhancing the power density of carbon materials are important aspects of developing carbon materials for advanced supercapacitors (Chen X. et al., 2014; Han et al., 2014; Yang and Zhou, 2017). Since the capacity of carbon materials depends on the amounts of electrostatic charges stored at the interfaces of the electrodes and electrolytes, a lot of researches have been done to design the desired carbon materials for ions storage. Tailoring the hierarchical pore structure to match the appropriate electrolyte ions is an effective method to achieve large capacitance, efficient ion diffusion, and charge transfer (Zhu et al., 2011; Fang et al., 2012; Chen et al., 2016, 2017; Yu et al., 2016; Liu et al., 2017; Shi et al., 2017, 2019). 
In recent years, some brilliant materials (Li et al., 2015, 2019a; Han et al., 2016; Cao et al., 2017; Lv et al., 2017a,b; Xu B. et al., 2017; Zhou and Hu, 2017; Zhou et al., 2018), including carbon nanotubes, grapheme, and their derivatives, have been widely explored and utilized because of their excellent thermal stability, remarkable tenacity, and high strength (Wen et al., 2016; Zhu et al., 2017; Liu et al., 2018). However, there are some obstacles in applying these materials as electrode materials for supercapacitors. The charge/discharge processes are directly affected by ionic kinetics, obviously under harsh charge/discharge conditions (current density higher than $10 \mathrm{~A} \mathrm{~g}^{-1}$ ) (An et al., 2001; Ra et al., 2009; Gao et al., 2012; Hahm et al., 2012; Sun et al., 2013; Zhu et al., 2016a). For instance, a capacitance of $362.2 \mathrm{~F} \mathrm{~g}^{-1}$ is obtained for the treated fullerene/graphite carbon electrodes at the low current density. However, when the current density increases to fifty times, the capacitance drops rapidly to half of the original value (Chen $Z$. et al., 2014; Zheng et al., 2015; Xu M. et al., 2017; Chen et al., 2018; Li et al., 2019b). It is not hard to note that increasing energy density under high power density of carbon electrodes is a great challenge. To the best of our knowledge, the poor performance in large current condition is associated with the sluggish ion migration in pores (Ye et al., 2019). It is difficult to keep good performances during charge/discharge processes without reasonable pore design. And electronic conductivity and ionic kinetics in electrodes can also be improved through regulating intrinsical structures of carbon materials (RaymundoPiñero et al., 2011; Mun et al., 2013; Zhu et al., 2016b). In general, internal pores affect ion transport while external pores are related to ion diffusion from electrolyte. Particularly, the reduced electrode potential caused by low access to ion in pores is the main impediments under large current (Presser et al., 2011; Gao et al., 2013; Wang et al., 2015). In order to explain the importance of the pores in carbon electrode theoretically, the transmission line model is employed to imitate the charge reserve characteristic of different pore shapes, showing that porous electrodes are conducive to charge accumulation (Black and Andreas, 2010; Zhang et al., 2017). The results are proposed that electrodes with multiscale pores are beneficial to ion diffusion, which is the key to obtain large capacitance at ultra-high current density. Micropores larger than $0.7 \mathrm{~nm}$ are found to be used as electrolyte reservoirs to store ions (Péan et al., 2014). Under the effect of the electric field, due to the shortened length of the ion diffusion, the accumulated ions can diffuse into the nearby micropores in a shorter time than the ions in the electrolyte. And pores larger than $2 \mathrm{~nm}$ assist to decrease the ion confinement in the micropores, significantly enhancing the effective self-diffusion coefficient of the ions, which is advantageous for obtaining excellent rate performance (Forse et al., 2017). Therefore, a better understanding of the relationship between pore structure and capacitance performance is essential to fabricate hierarchical porous structure in carbon materials. It will accelerate ion kinetics to obtain carbon electrode materials with high ion transfer rate, which can achieve high energy storage under high current density. In order to obtain highperformance carbon electrode materials with hierarchical pore stricture, the strategy for fast ion transfer leaded by economical and efficient synthesis has been focused as main interests (Chen et al., 2013; Wang et al., 2017).

The main approaches to design hierarchical porous carbon materials are as following: (1) pores or defects generated on the surface or matrix through physical or chemical activation; (2) pores created by multidimensional carbon coupling/selfassembly; (3) adjustment of microstructures by means of templates; (4) pores congregated by the polymerization of nanoscale building blocks (Kang et al., 2015; Ni and Li, 2016; Yin et al., 2018; Kong et al., 2019). In these methods above, the size and shape of the pore array can be well-adjusted by changing the characteristics of template. However, the instability of nanoscale templates under high temperature or solvent treatment limits its extensive application (Fuertes et al., 2005; $\mathrm{Lv}$ et al., 2012). The process of multi-dimensional carbon coupling with the guest species and polymerization through building blocks is also tedious. Consequently, a wide range of biomass precursors combined with efficient activation methods are properly employed to prepare high-performance hierarchical porous carbon materials. Hierarchical pores in the surface would be beneficial to the transport of ions between adjacent layers, maximizing the reachable surface area. Due to the resulted multiple porous structure, the advantageous electronegativity also shows that carbon materials possess superior electrochemical performance in energy storage (Gao et al., 2019; Cai et al., 2020). Durian is widely distributed in the Southeast Asia region and around 90,000 metric tons of durian wastes were generated annually. Note that the advantages of durian shells are easy availability, cheap and high economic value. The reason of choosing durian shell as precursor to prepare carbon electrode can be attributed to main two reasons: (1) the hierarchical porous structure of the durian shell shows fast mass transfer channel, carbon material derived from durian shell precursor maintain the inherit structure features, possessing the fast ion/electron transport rate; (2) durian shell contain some heteroatoms, which can introduce defects during carbonization process, increasing the wettability of the surface of the carbon material, and promoting the contact of electrolyte area. In this work, a multi-stage porous carbon material (DSPC) is successfully obtained through the pre-carbonization and activation of the durian shell. By adjusting the activation temperature, the degree of porosity of the DSPC can be well-manipulated. The DSPC exhibits typical hierarchical porosity, reasonable aperture distribution, and high specific surface area. Surprisingly, symmetric supercapacitors based on DSPC electrodes deliver large capacity and fast ion transfer characteristics in aqueous electrolyte and organic electrolyte.

\section{EXPERIMENTAL SECTION}

\section{Preparation of DSPCs}

Firstly, the durian shell is cleaned several times by distilled water to remove some impurities and put into an oven under $120^{\circ} \mathrm{C}$ and for $24 \mathrm{~h}$. Secondly, the dried durian shell is directly transferred to a tubular furnace in Ar atmosphere. The carbonized temperature is set to $400^{\circ} \mathrm{C}$ for $2 \mathrm{~h}$ with a heating rate of $3^{\circ} \mathrm{C} \mathrm{min}^{-1}$. The obtained sample is marked as DSPC. Finally, the DSPC 
is manufactured with surface defects to obtain hierarchical channels. DSPC is mixed and grinded with $\mathrm{KOH}$ of $4: 1$ mass ratio. In order to explore the most appropriate the ratio of $\mathrm{KOH}$ to $\mathrm{C}$, the corresponding electrochemical results are obtained in Figure S1. They are divided into five parts for subsequent activation and immediately transferred to a tubular furnace for generating surface defects. The temperature is 600, 700, 800, 900, and $1,000^{\circ} \mathrm{C}$ for $1 \mathrm{~h}$. All the samples after being calcined are added $6 \mathrm{M} \mathrm{HCl}$ until $\mathrm{pH}=7$. All samples are then washed to neutral and dried in an oven. The carbon materials activated under different temperature are denoted as DSPC-600, DSPC700, DSPC-800, DSPC-900, and DSPC-1000, respectively.

\section{Material Characterization}

The structure of DSPCs are explored by X-ray diffraction (XRD) with a scan rate of $10^{\circ} \mathrm{C} \mathrm{min}^{-1}$ by using $\mathrm{K} \alpha$ radiation of $\mathrm{Cu}(\lambda=0.1542 \mathrm{~nm})$. Degree of graphitization is measured through Raman spectroscopy (DXR, Thermo-Fisher Scientific). The morphology of all the samples are investigated by fieldemission scanning electron microscopy (FESEM, JSM-7600F, JEOL) and transmission electron microscopy (JEM-2010F at $200 \mathrm{kV}, \mathrm{JEOL})$. The specific surface area is acquired from the $\mathrm{N}_{2}$ adsorption-desorption isotherms and is obtained by the Brunauere Emmette Teller (BET) equation. The chemical bond of DSPCs are collected from X-ray photoelectron spectrometer (XPS) instrument.

\section{Electrochemical Characterization}

All the samples are measured on a three-electrode system using aqueous electrolyte and on two-electrode system using aqueous electrolyte and organic electrolyte for determining their capacitive performance.

For three-electrode aqueous electrolyte system, every carbon samples are grinded with carbon black and polytetrafluoroethylene (PTFE) with a weight ratio of 8:1:1 loaded on nickel foam. The electrode plates are then dried in a vacuum at $80^{\circ} \mathrm{C}$ for $12 \mathrm{~h}$. Pieces are pressed under $10 \mathrm{MPa}$ for $1 \mathrm{~min}$. The mass load of each electrode plate is $2.2 \mathrm{mg} / \mathrm{cm}^{2}$. Platinum electrode is selected as the counter electrode, and a saturated calomel electrode is selected as the reference electrode. The electrolyte is $6 \mathrm{M} \mathrm{KOH}$.

For two-electrode aqueous electrolyte system, every carbon samples are grinded with carbon black and polytetrafluoroethylene (PTFE) with a weight ratio of $8: 1: 1$ loaded on nickel foam. The electrode plates are then dried in a vacuum at $80^{\circ} \mathrm{C}$ for $12 \mathrm{~h}$. Pieces are pressed under $10 \mathrm{MPa}$ for $1 \mathrm{~min}$. The electrolyte is $6 \mathrm{M} \mathrm{KOH}$. CR2016-type coin cells are assembled accordingly. Electrochemical test begins after several hours.

For two-electrode organic electrolyte system, each carbon samples are grinded with carbon black and polyvinylidene fluoride (PVDF) with a weight ratio of 8:1:1 loaded on Al foils. The electrode plates are then dried in a vacuum at $120^{\circ} \mathrm{C}$ for $12 \mathrm{~h}$. CR2016-type coin cells are assembled inside the Braunglovebox with argon atmosphere $\left(<0.1 \mathrm{ppm}\right.$ of both $\mathrm{O}_{2}$ and $\left.\mathrm{H}_{2} \mathrm{O}\right)$. Galvanostatic charge/discharge (GCD) tests are carried out between 0 and $2.7 \mathrm{~V}$ by an Arbin BT2000 instrument. The cyclic voltammetry (CV) tests are carried out at different scan rates on a Solartron 1470 Multistat system. The electrochemical impedance spectroscopy (EIS) measurements are performed using an electrochemical workstation.

The gravimetric capacitance is calculated from the CV curves and discharge curves, according to the formula:

$$
C s=\frac{I \Delta t}{m \Delta V}
$$

where $C s$ is the specific capacitance and $\Delta V$ is the voltage drop. $\Delta t$ is the discharging time. $I / m$ is referred to the current density.

The power density and energy density of the devices are obtained thorough using the following equations:

$$
\begin{aligned}
& P=\frac{\triangle V \times I}{2 \times m} \\
& E=\frac{P * t}{3600}
\end{aligned}
$$

Here, $\Delta V$ is referred to the discharge voltage, $I$ is referred to the discharge current, $t$ is referred to the discharge time and $\mathrm{m}$ is referred to the total mass of active materials, respectively.

\section{RESULTS AND DISCUSSION}

SEM and TEM images of hierarchical porous carbon materials prepared from the durian shell are shown in Figure 1 and Figure S2. It is obvious that the pores are hardly manufactured at $600^{\circ} \mathrm{C}$. When the temperature is increased to $700^{\circ} \mathrm{C}$, the pore structure in the carbon material is gradually generated, and an uneven structure is produced on the surface. As the temperature is further risen up to $800^{\circ} \mathrm{C}$, the pore structure formed in the carbon material are widely ranged and the structure is uniform. When the temperature is raised to $900^{\circ} \mathrm{C}$, the defect of the surface is degenerated and destroyed, and the pore structure of the material is broken. As the temperature is kept at $1,000^{\circ} \mathrm{C}$, the structure of hierarchical pores is collapsed more severely, and the surface defect structure is difficult to be observed. According to TEM images, an amorphous dominated porous structure and defect architecture are further revealed.

The XRD measurement of all samples are exhibited in Figure 2A. The broad peaks located at $23^{\circ}$ and inconspicuous peaks centered at $43^{\circ}$ are perceived, indicating that all the samples are in amorphous constructions maintained few regions of crystallinity (Hou et al., 2015, 2017; Ge et al., 2018; Zou et al., 2018; Wu et al., 2019). More detailer pore structures of the samples are collected by the nitrogen adsorption/desorption measurements in Figures 2B,C. The isotherms of all DSPCs exhibit a combination of I-type and IV-type isotherm characteristics at a relative pressure $>0.4$, suggesting that both micropores and mesopores exist. Notably, the specific surface area of DSPC-600, DSPC-700, DSPC-800, DSPC-900, and DSPC-1000 are 1006, 1217, 2535, 2602, and $899 \mathrm{~m}^{2} \mathrm{~g}^{-1}$, respectively. When the temperature is increased slightly, the surface area of micropores and mesoporous increase. As the increase of temperature, the proportion of micropores decreases while the proportion of mesopores increases according 


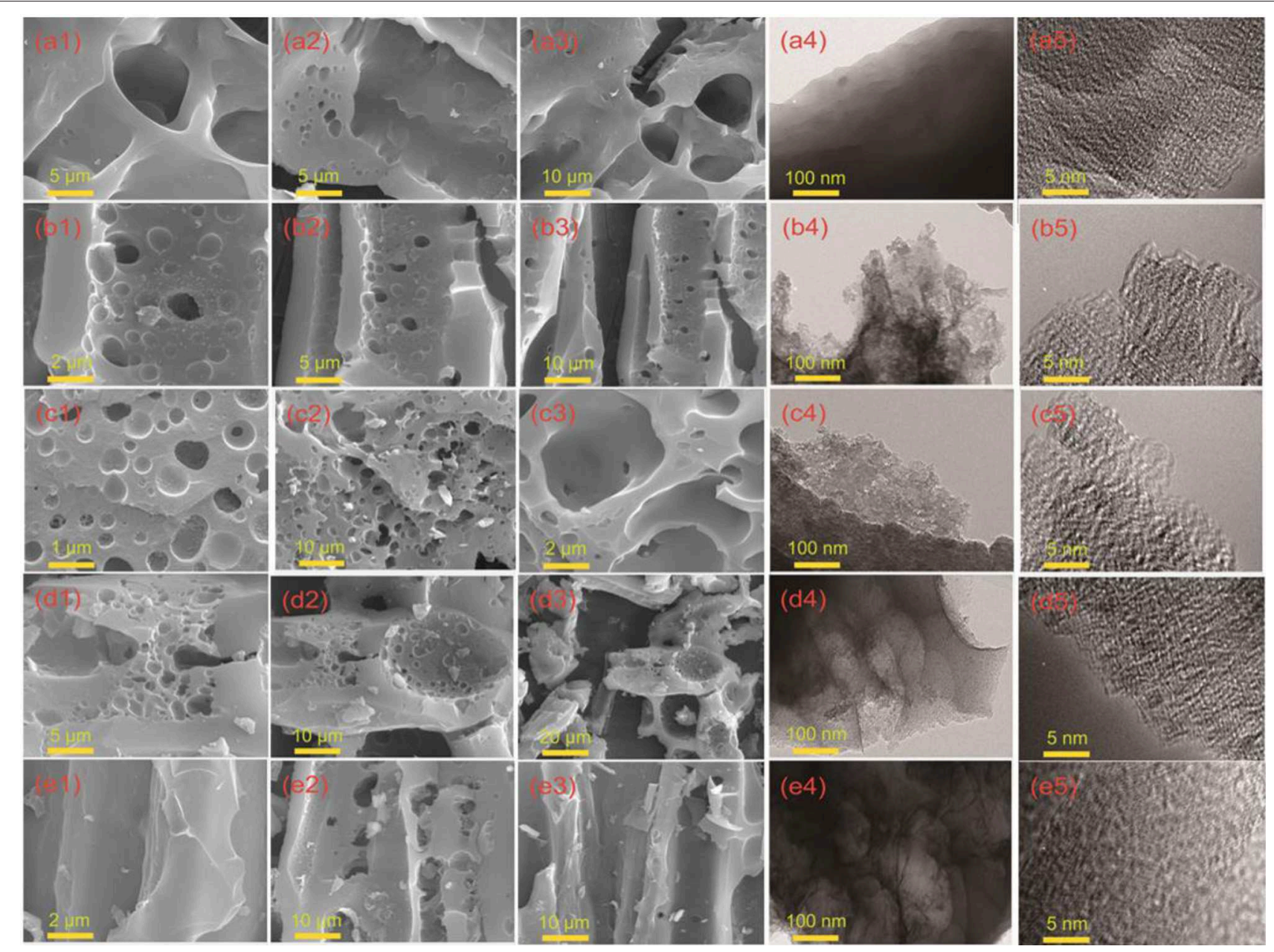

FIGURE 1 | Morphology and structure characterization of DSPCs. (a1-a3) SEM image of DSPC-600, (a4,a5) TEM and HRTEM image of DSPC-600. (b1-b3) SEM image of DSPC-700, (b4,b5) TEM and HRTEM image of DSPC-700. (c1-c3) SEM image of DSPC-800, (c4,c5) TEM and HRTEM image of DSPC-800. (d1-d3) SEM image of DSPC-900, (d4,d5) TEM and HRTEM image of DSPC-900. (e1-e3) SEM image of DSPC-1000, (e4,e5) TEM and HRTEM image of DSPC-1000.

to the higher platform and lower starting angles of the lowpressure areas of DSPC-900 in Figure 2B. The plots of pore size distributions are revealed the porous structure for all the samples. Furthermore, wide existence of nanopores in plots of pore size distributions illustrates hierarchical porous structure of DSPC800 . Risen from $800^{\circ} \mathrm{C}$ to $1,000^{\circ} \mathrm{C}$, the distribution of micropores gradually decreases and the distribution of mesopores becomes larger. In general, as depicted in Figure 2C, the DSPC-800 exhibits a hierarchically aperture and porosity structure. The Raman spectra of the acquired samples are deconvoluted into two peaks including $G$ peak and $D$ peak, and the intensity ratio of $\mathrm{G}$ band vs. D band (IG/ID) can be used to describe the degree of graphitization. The $\mathrm{G}$ peak is induced by Sp2-hybrid carbon and D peak is related to the disordered graphite structure. As shown in Figure 2D, the values of IG/ID of all samples are 1.65, $2.52,6.6,2.06$, and 1.59, respectively, demonstrating the high graphitization degree of DSPC-800. Note that Sp2 hybridizationdominant carbon is widely employed to improve ion transfer rate and efficient energy storage because of large graphitization, high electrical conductivity, and thermal stability. Benefiting from high value of IG/ID in DSPC-800 superior kinetics performance during chare/discharge process could be delivered. Furthermore, the surface chemical environment of DSPC-800 is determined in details by XPS analysis. Carbon content in the DSPC-800 is 91.02 at\%, and the oxygen content of the DSPC-800 is 8.98 at $\%$. As shown in Figure $2 \mathrm{E}$, the $\mathrm{C} 1$ s profiles is divided into four peaks of $\mathrm{COOH}(290 \mathrm{eV}), \mathrm{C}=\mathrm{O}(286.7 \mathrm{eV}), \mathrm{C}-\mathrm{O}(285-286 . \mathrm{eV})$ and $\mathrm{C}=\mathrm{C} / \mathrm{C}-\mathrm{C}(284.6 \mathrm{eV})$. The high-resolution $\mathrm{O} 1 \mathrm{~s}$ spectra can be deconvoluted into three peaks located at 531.0, 533, and $536 \mathrm{eV}$, which is corresponding to the $\mathrm{C}=\mathrm{O}, \mathrm{C}-\mathrm{O}$, and carbonyl (Figure 2F), respectively (Yang et al., 2019; Zhu et al., 2020).

The capacitive performance of DSPCs are firstly measured in 6.0 M KOH/ $\mathrm{H}_{2} \mathrm{O}$ electrolyte by using a three-electrode system. The CV curves of all the samples exhibit quasi-rectangular appearance at the scan rate of $5-100 \mathrm{mV} \mathrm{s}^{-1}$, implying an ideal capacitive characteristic in Figures 3a1-e1. The DSPC-800 shows the largest area of CV curves among all the samples, suggesting outstanding charge storage feature. In addition, according to the 
A

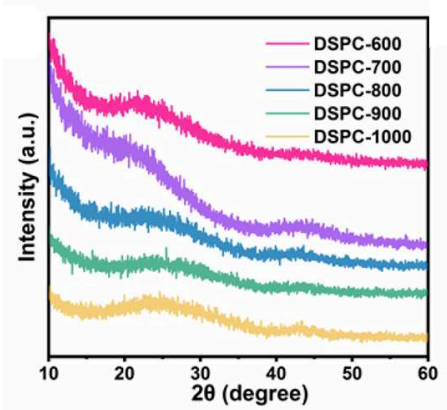

D

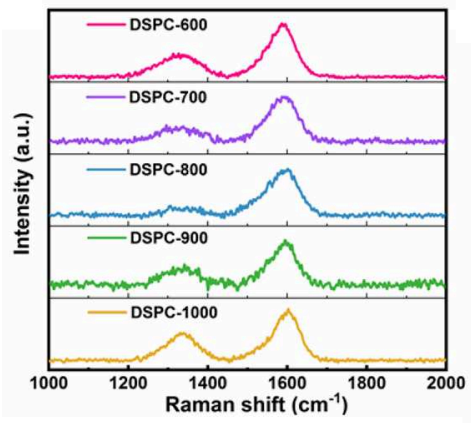

B

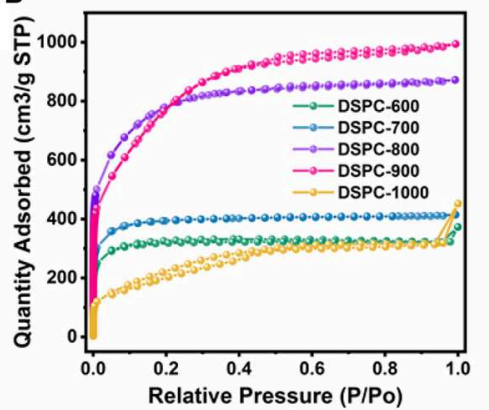

E

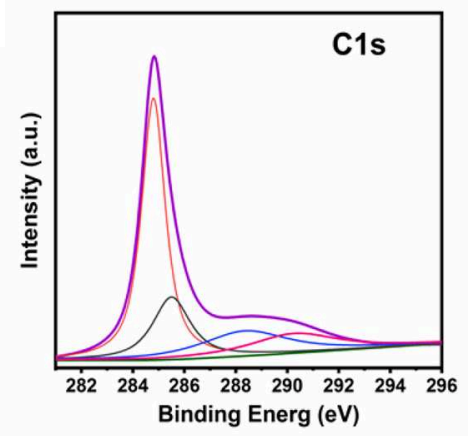

C

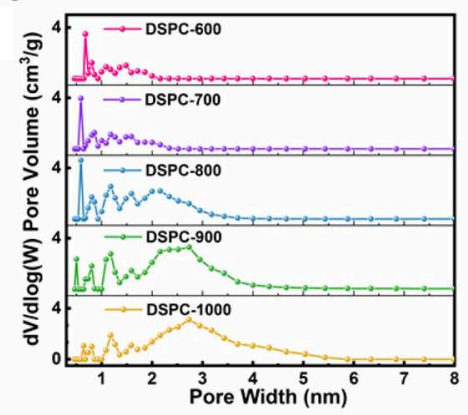

$\mathbf{F}$

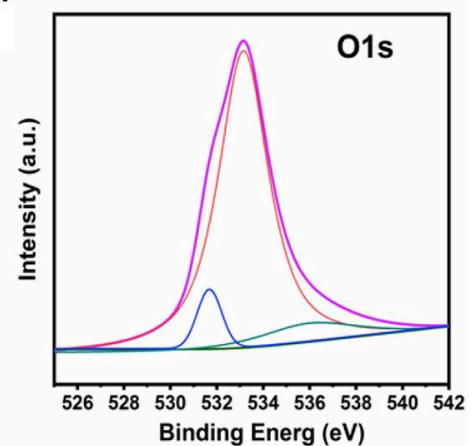

FIGURE 2 | (A) XRD patterns, (B) N2 adsorption-desorption isotherms, (C) Pore size distribution, and (D) Raman spectrum of DSPCs. (E,F) high-resolution XPS C1s and O1s spectra for DSPC-800.

results in Figure S1, the ratio of $\mathrm{KOH}$ to $\mathrm{C}$ should be selected as 4: 1 rather than 3:1 or 5:1. As displayed in Figure 3c2, the GCD curve of DSPC-800 presents the longest discharge time among the five samples at the current density of $10 \mathrm{~A} \mathrm{~g}^{-1}$, suggesting the largest capacitance among all the samples. Based on the results of GCD curves, the specific capacitances of DSPC-600, DSPC700, DSPC-800, DSPC-900, and DSPC-1000 are 239.5, 336.6, $320.5,209.6$, and $186 \mathrm{~F} \mathrm{~g}^{-1}$ at a current density of $0.5 \mathrm{~A} \mathrm{~g}^{-1}$ (Figures 3a2,b2,d2,e2), exhibiting that the specific capacitances of DSPCs are 194.1, 249.1, 253.1, 158.9, and 159 at $5 \mathrm{~A} \mathrm{~g}^{-1}$, respectively. Impressively, the specific capacitances of DSPCs are $130.4,93.7,154.75,50$, and $60 \mathrm{~F} \mathrm{~g}^{-1}$ at a current density of $50 \mathrm{~A} \mathrm{~g}^{-1}$, separately. The capacitive performances of DSPCs are subsequently evaluated in $6.0 \mathrm{M} \mathrm{KOH} / \mathrm{H}_{2} \mathrm{O}$ electrolyte using a two-electrode system. The CV curves of the device are listed in Figure 3c3, in which the quasi-rectangular appearance of DSPC800 is kept even at the scan rate of $100 \mathrm{mV} \mathrm{s}^{-1}$, demonstrating an excellent rate performance. This result is further illustrated by the GCD curves at different current densities in Figures 3a3-e3. When the current density is $0.5 \mathrm{~A} \mathrm{~g}^{-1}$, the specific capacitances of DSPCs devices are determined as 56.1, 60.9, 82, 52, and $46.3 \mathrm{~F}$ $\mathrm{g}^{-1}$, respectively, corresponding to the specific capacitances of $45.1,42.7,63.3,33$, and $32 \mathrm{~F} \mathrm{~g}^{-1}$ at $5 \mathrm{~A} \mathrm{~g}^{-1}$, respectively. As the current density increases to $20 \mathrm{~A} \mathrm{~g} \mathrm{~g}^{-1}$, specific capacitances of $30.2,25.1,32.1,9.3$, and $12 \mathrm{~F} \mathrm{~g}^{-1}$ are achieved for DSPCs samples. The highest specific capacitance of DSPC-800 is attributed to hierarchical porous structure, which can bring numerous active sites for the charge storage in Figures 3a4-e4.
All the samples are further tested in organic electrolyte in two-electrode devices based on DSPCs. The device of DSPC-600 measured in organic electrolyte is shown an obvious polarization, which is attributed to sluggish ion kinetics behavior of the electrode (Figure 4a1). The low energy density of the device of DSPC-600 is $0.01 \mathrm{Wh} \mathrm{kg}^{-1}$, which is suggested andante charge transport and ion diffusion in large current density shown in Figure 4a3. The low capacity can be attributed to the less surface defects and low conductivity due to the low activation temperature. The other four devices are shown with a quasi-rectangular shape CV curve that can be interpreted as the presence of higher surface defects. The DSPC-800 device shows a highly symmetric shape, suggesting great electrochemical reversibility (Figures $\mathbf{4 b 1 - e 1 )}$ ). It is important to noted that the device of DSPC-800 exhibits smallest IR drop at $10 \mathrm{~A} \mathrm{~g}^{-1}$, implying that surface defect structure with relatively high hierarchical aperture and porosity can facilitate transfer rate of the electrolyte ions and decrease the ionic diffusivity resistance. This result is further illustrated by the GCD curves at different current densities (Figures 4a3-e3). The energy density of all the devices are $21.5,24.3,27.7,22$, and $10.4 \mathrm{Wh} \mathrm{kg}^{-1}$ at a power density of $675 \mathrm{~W} \mathrm{~kg}-1$, respectively. Furthermore, energy densities of $0.6,20.7,21.5,16.4$, and 8.4 Wh $\mathrm{kg}^{-1}$ can be obtained for related samples at a power density of $6,750 \mathrm{~W} \mathrm{~kg}^{-1}$. Surprisingly, even at $67,500 \mathrm{~W} \mathrm{~kg}^{-1}$, the device of DSPC-800 can also deliver an energy density of $4.2 \mathrm{Wh} \mathrm{kg}^{-1}$. The highest energy density of the devices with DSPC-800 as electrode are attributed to multiple pores 


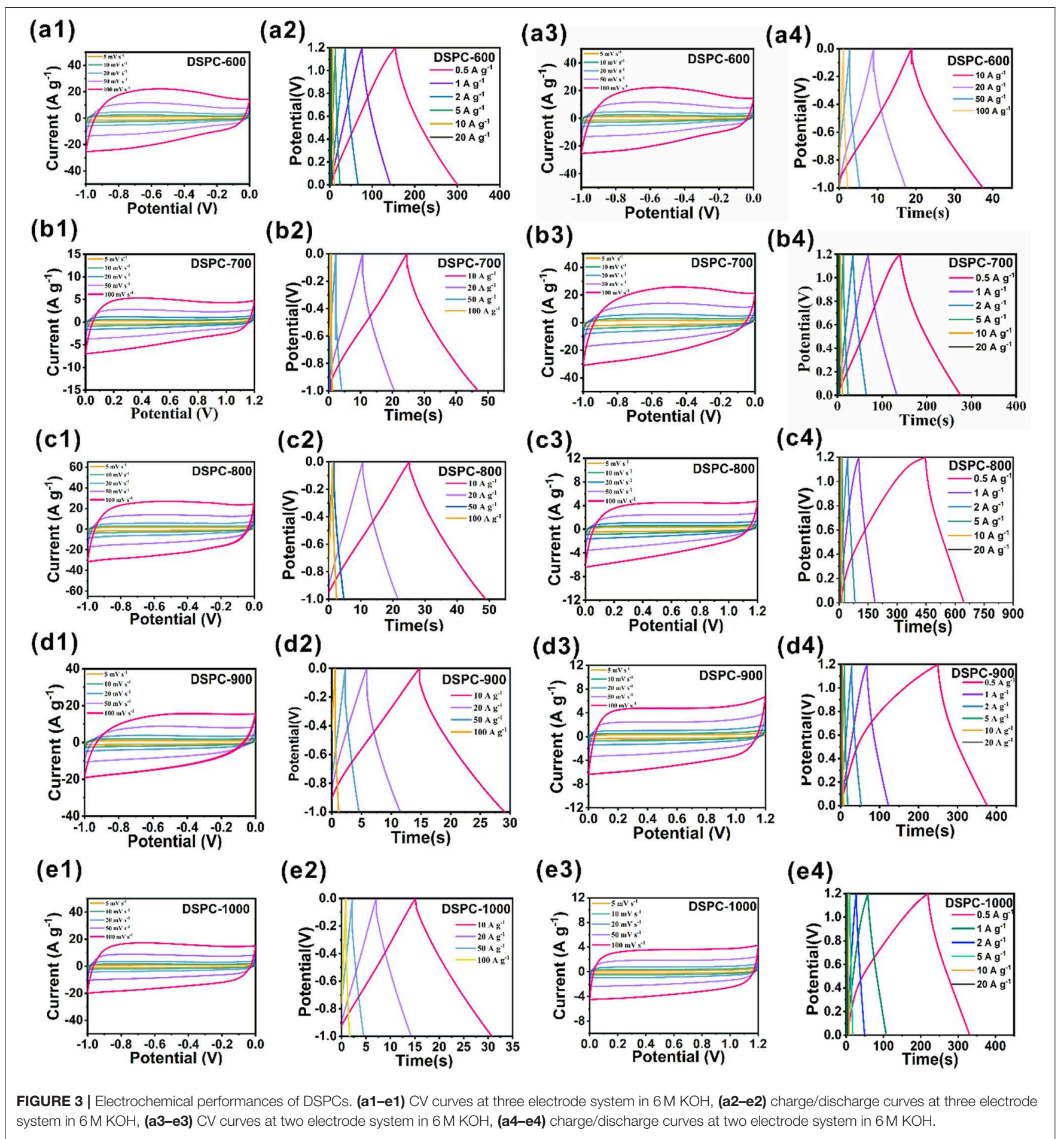

that can supply numerous active sites for the charge storage in Figure 4. It is clear that hierarchical porous structure can effectively improve the electrochemical properties of materials in both aqueous electrolyte and organic electrolyte system, suggesting that the introduction of hierarchical porous structure is significant.

A number of electrochemical measurements including cycling stability and electrochemical impedance spectroscopy (EIS) are carried out to investigate the electrochemical performance of DSPCs. Low resistance and rate capability are determined by the efficiency of charge transport and ion diffusion in aqueous electrolyte system ( $\mathrm{Xu}$ et al., 2014). The contact resistance between the electrode material and the electrolyte also affects resistance (Rs). Because the ion transfer characteristics of these synthesized materials are different, Rs is generally different (Jin et al., 2018a,b; Nawwar et al., 2019; Wang and Cui, 2019; 
(a1)

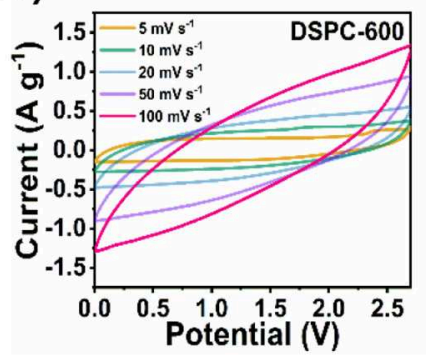

(b1)

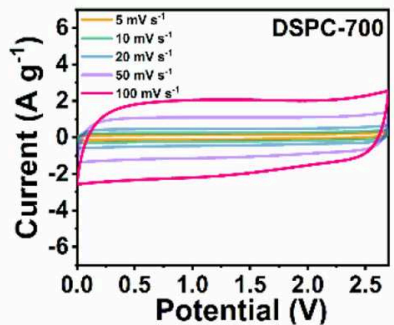

(c1)

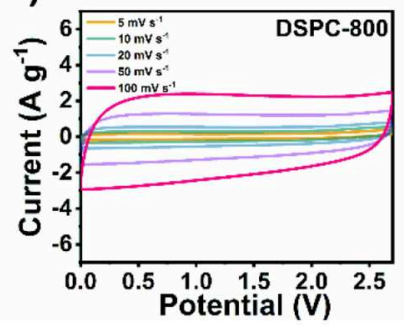

(d1)

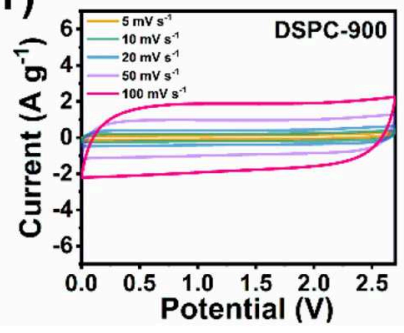

(e1)

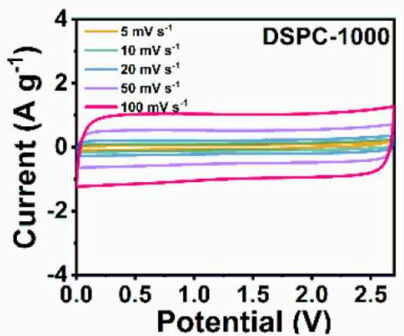

(a2)

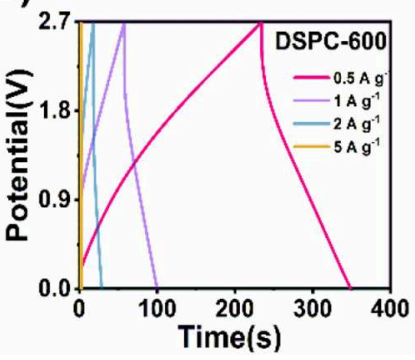

(b2)

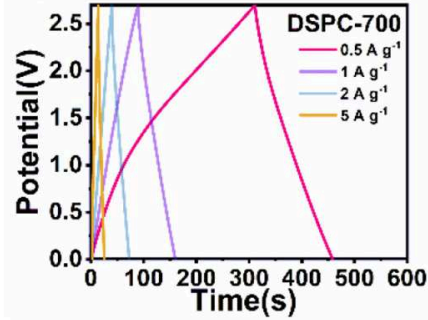

(c2)

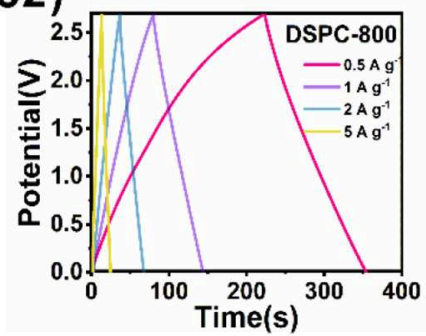

(d2)

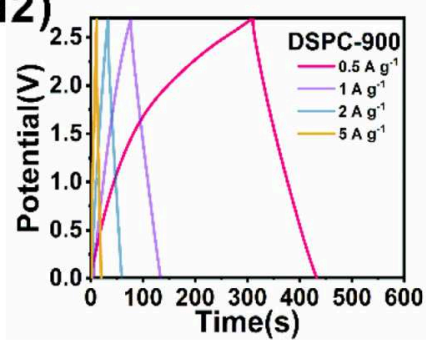

(e2)

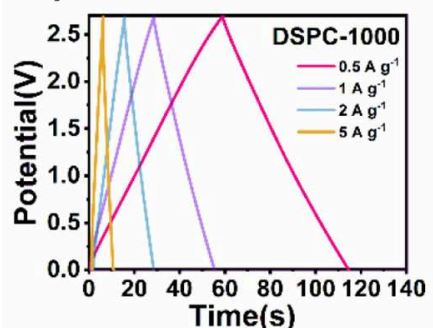

(a3)

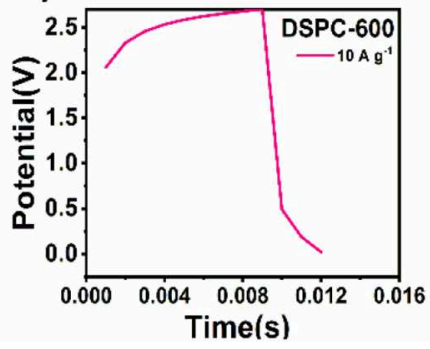

(b3)

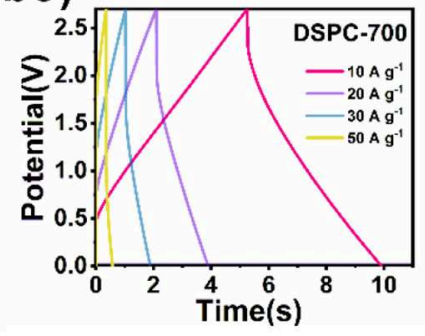

(c3)

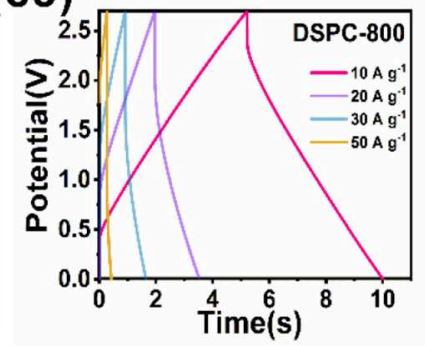

(d3)

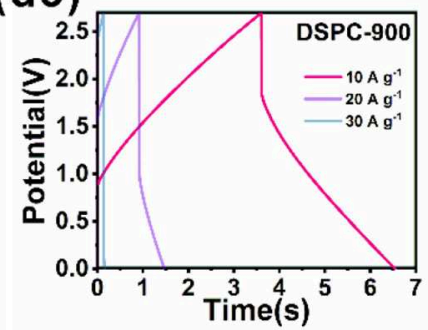

(e3)

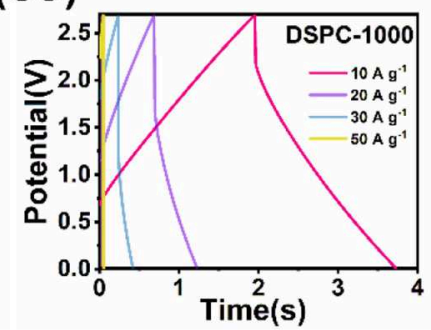

FIGURE 4 | Electrochemical performances of DSPCs. (a1-e1) CV curves at two electrode system in organic electrolyte, (a2-e2) charge/discharge curves at two electrode system in organic electrolyte, (a3-e3) charge/discharge curves at two electrode system tested in large current in organic electrolyte. 
Yuan and Lu, 2019). The surface defect of DSPCs is critical for achieving such high specific capacitance. EIS analysis is revealed that DSPC-800 exhibits an ignorable combined series resistance, which is greater than other samples. The steep slope of the Nyquist plot in the low frequency domain indicates fast ion transport in the surface defect electrode. The lack of semicircle of DSPC-800 in the middle frequency domain further suggests that the charge transfer resistance (Rct) at the electrode/electrolyte interface is negligible (Figure 5A). In organic electrolyte system, DSPC-800 also exhibits a small resistance, which is similar to the results in aqueous electrolyte system in Figure 5A. The large resistance of DSPC-600 is correlated with previous results. Bode phase diagram also discloses some important information in Figures 5B,E. Firstly, the $0.1 \mathrm{~Hz}$ phase angle is approximately the ideal phase angle, that is $-90^{\circ}$, and the ideal phase angle is closed to the ideal capacitive behavior. Secondly, the characteristic frequency $\left(\mathrm{f}_{0}\right)$ is corresponded to a $-45^{\circ}$ phase angle. The characteristic time constant can be expressed as: $\tau_{0}=1 / \mathrm{f}_{0}$. The smaller $\tau_{0}$ represents the faster frequency response and $\tau_{0}$ is related to the rate characteristics. Bode phase diagram is revealed that surface defect is contributed to ion diffusion (Figures 5B,E). A high capacity residue of $88.71 \%$ can be acquired for DSPC-800 in aqueous electrolyte system at $20 \mathrm{~A} \mathrm{~g}^{-1}$ after 10,000 cycles. The capacity retentions of other devices are $82.9,84.75,57.64$, and $72.98 \%$ in aqueous electrolyte system at $20 \mathrm{~A} \mathrm{~g} \mathrm{~g}^{-1}$ (Figure 5C), respectively. DSPC-800 is also preserved good cycle performance in organic electrolyte system after several thousand times (Figure 5). Note that carbon materials with hierarchical porous structure assembled devices can deliver remarkable performance and show practical applications (Figures 5G-I).

\section{CONCLUSION}

Here, defect strategy is demonstrated as an effective method to construct hierarchical porous structure with fast ion transport property. The hierarchical porous structure provides larger surface area for the infiltration of electrolyte for ions transport into interior multiple pores. Consequently, hierarchical porous structure can optimize ion kinetics by affecting ion transport of internal pores and ion diffusion of external pores from electrolyte, especially at large current density. Due to the hierarchical porous structure tuned high ionic conductivity and optimized ionic kinetics of electrodes, the power performance is impressive, delivering excellent gravimetric capacitance both in aqueous and organic electrolyte. This work provides a bright and effective approach to fabricate the carbon materials with hierarchical porous structure. Surprisingly, it has been successfully applied in energy storage under large current density. Considering the advantages of low cost and abundance, hierarchical porous carbon is extremely promising for the development of advanced electrode materials in super capacitors.
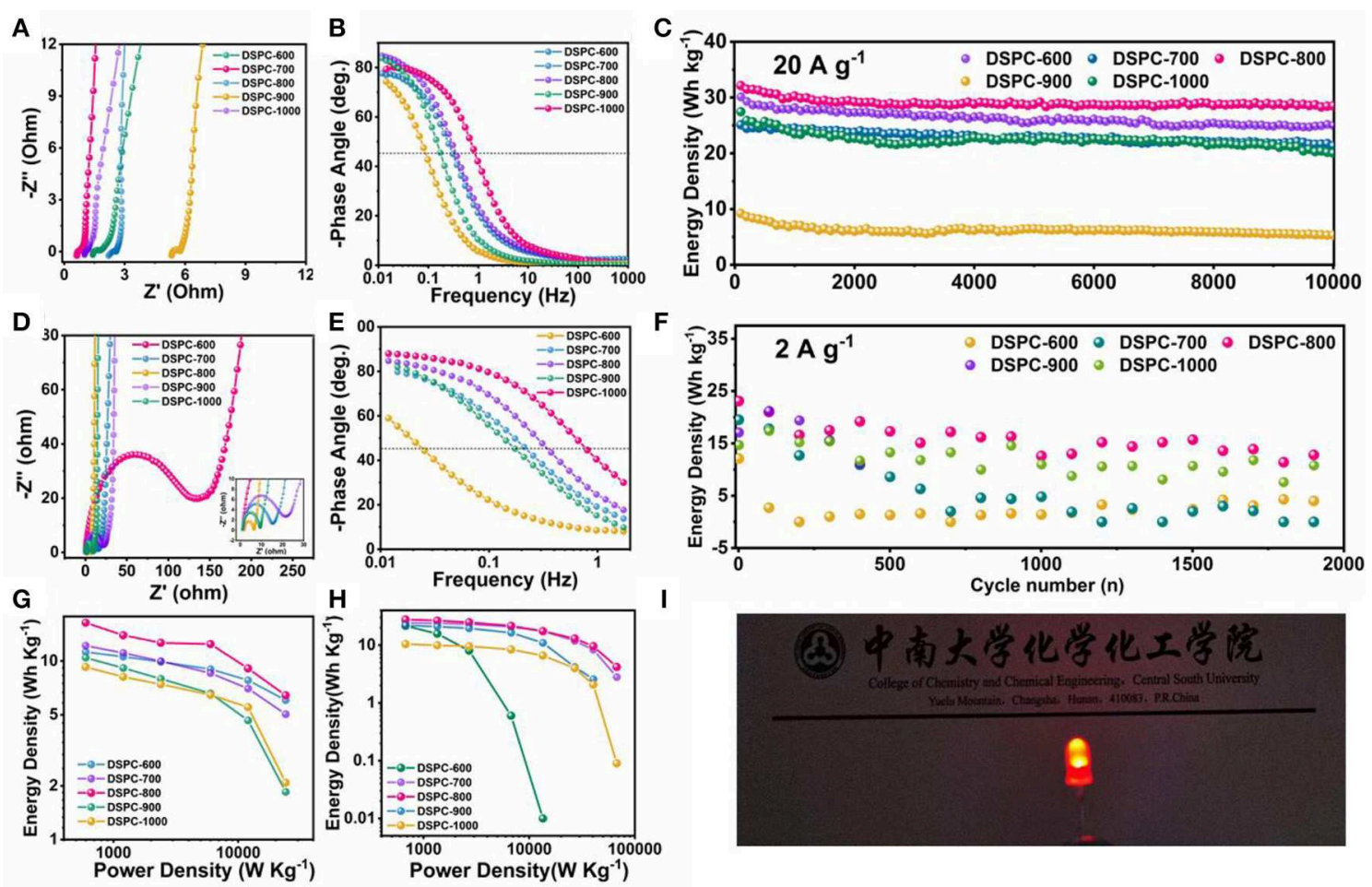

FIGURE 5 | (A) EIS analysis in $6 \mathrm{M} \mathrm{KOH}$, (B) Bold plot in $6 \mathrm{M} \mathrm{KOH}$, (C) cycle performance in $6 \mathrm{M} \mathrm{KOH}$, (D) EIS analysis in organic electrolyte, (E) Bold plot in organic electrolyte, (F) cycle performance in organic electrolyte, $\mathbf{( G , H ) ~ R a g o n ~ p l o t ~ i n ~} 6 \mathrm{M} \mathrm{KOH}$ and organic electrolyte, (I) optical picture of device of DSPC-800 in organic electrolyte. 


\section{DATA AVAILABILITY STATEMENT}

The raw data supporting the conclusions of this article will be made available by the authors, without undue reservation, to any qualified researcher.

\section{AUTHOR CONTRIBUTIONS}

PC and GZ designed the research, performed the experiments, and made contributions to the acquisition, analysis, and interpretation of data for the work. KZ, XD, and BW carried out the partial experimental characterization. $\mathrm{HH}$ and $\mathrm{XJ}$ revised this article and approved of the version to be published. All authors incorporated in the discussion of experimental results.

\section{REFERENCES}

An, K. H., Kim, W. S., Park, Y. S., Moon, J. M., Bae, D. J., Lim, S. C., et al. (2001). Electrochemical properties of high-power supercapacitors using single-walled carbon nanotube electrodes. Adv. Funct. Mater. 11, 387-392. doi: 10.1002/16163028(200110)11:5andlt;387::AID-ADFM387andgt;3.0.CO;2-G

Béguin, F., Presser, V., Balducci, A., and Frackowiak, E. (2014). Carbons and electrolytes for advanced supercapacitors. Adv. Mater. Weinheim. 26, 2219-2251. doi: 10.1002/adma.201304137

Black, J., Andreas, H. J. (2010) Pore Shape Affects Spontaneous Charge Redistribution in Small Pores. Phys. Chem. C. 114, 12030-12038. doi: $10.1021 /$ jp $103766 \mathrm{q}$

Cai, P., Zou, Y., Zou, G., Hou, H., and Ji, X. (2020). Quinone/ester-based oxygen functional groups enhanced full carbon Li-ion capacitor. Nanoscale doi: 10.1039/C9NR10339B. [Epub ahead of print].

Cao, D., Yin, C., Shi, D., Fu, Z., and Zhang, J. (2017). Cubic perovskite fluoride as open framework cathode for Na-ion batteries. Adv. Funct. Mater. 28:1701130. doi: 10.1002/adfm.201701130

Chen, D., Tian, J., Liu, C., and Du, M. (2016). A bracket approach to improve the stability and gas sorption performance of a metal-organic framework via in situ incorporating the size-matching molecular building blocks. Chem. Commun. 52, 8413-8416. doi: 10.1039/c6cc02359B

Chen, D., Tian, J., Wang, Z., Liu, C., Chen, M., and Du, M. (2017). An anionic $\mathrm{Na}$ (I)-organic framework platform: separation of organic dyes and postmodification for highly sensitive detection of picric acid. Chem. Commun. 53, 10668-10671. doi: 10.1039/C7CC06073D

Chen, J., Li, L., Wu, L., Yao, Q., Yang, H., Liu, Z., et al. (2018). Enhanced $\begin{array}{lllllllllll}\text { cycle stability of } & \mathrm{Na} & 0.9 & \mathrm{Ni} & 0.45 & \mathrm{Mn} & 0.55 & \mathrm{O}_{2} & \text { through tailoring O3/P2 }\end{array}$ hybrid structures for sodium-ion batteries. J. Power Sour. 406, 110-117. doi: 10.1016/j.jpowsour.2018.10.058

Chen, X., Lin, H., Chen, P., Guan, G., Deng, J., and Peng, H. (2014). Smart, stretchable supercapacitors. Adv. Mater. Weinheim. 26, 4444-4449. doi: 10.1002/adma.201400842

Chen, X., Qiu, L., Ren, J., Guan, G., Lin, H., Zhang, Z., et al. (2013). Novel electric double-layer capacitor with a coaxial fiber structure. Adv. Mater. Weinheim. 25, 6436-6461. doi: 10.1002/adma.201301519

Chen, Z., Xu, M., Du, B., Zhu, H., and Xie, T., Wang,W. (2014). Morphology control of lithium iron phosphate nanoparticles by soluble starch-assisted hydrothermal synthesis. J. Power Sour. 272, 837-844. doi: 10.1016/j.jpowsour.2014.09.019

Fang, Y., Luo, B., Jia, Y., Li, X., Wang, B., Song, Q., et al. (2012). Renewing functionalized graphene as electrodes for high-performance supercapacitors. Adv. Mater. Weinheim. 24, 6348-6355. doi: 10.1002/adma.201202774

Forse, A., Griffin, J., Merlet, C., Carretero-Gonzalez, J., Raji, A., Trease, N., et al. (2017). Direct observation of ion dynamics in supercapacitor electrodes using in situ diffusion NMR spectroscopy. Nat. Energy 2:16216. doi: 10.1038/nenergy.2016.216

\section{FUNDING}

This work was financially supported by the National Key Research and Development Program of China (2018YFC1901605), the National Postdoctoral Program for Innovative Talents (BX201600192), and the Hunan Provincial Science and Technology Plan (2017TP1001), Innovation Mover Program of Central South University (GCX20190893Y).

\section{SUPPLEMENTARY MATERIAL}

The Supplementary Material for this article can be found online at: https://www.frontiersin.org/articles/10.3389/fchem. 2020.00043/full\#supplementary-material

Fuertes, A., Lota, G., Centeno, T. A., and Frackowiak, E. (2005). Templated mesoporous carbons for supercapacitor application. Electrochim. Acta 50, 2799-2805. doi: 10.1016/j.electacta.2004.11.027

Gao, X., Jiang, F., Yang, Y., Zhang, Y., Zou, G., Hou, H., et al. (2019). Chalcopyritederived NaxMO2 ( $\mathrm{M}=\mathrm{Cu}, \mathrm{Fe}, \mathrm{Mn})$ cathode: tuning impurities for self-doping. ACS Appl. Mater. Inter. 12, 2432-2444. doi: 10.1021/acsami.9b17952

Gao, Y., Presser, V., Zhang, L., Niu, J., McDonough, J., Pérez, C., et al. (2012). High power supercapacitor electrodes based on flexible TiC-CDC nano-felts. J. Power Sources 201, 368-375. doi: 10.1016/j.jpowsour.2011.10.128

Gao, Y., Zhou, Y., Qian, M., He, X., Redepenning, J., Goodman, P., et al. (2013). Chemical activation of carbon nano-onions for high-rate supercapacitor electrodes Carbon N. Y. 51, 52-58. doi: 10.1016/j.carbon.2012.08.009

Ge, P., Hou, H., Cao, X., Li, S., Zhao, G., Guo, T., et al. (2018). Multidimensional evolution of carbon structures underpinned by temperatureinduced intermediate of chloride for Sodium-ion batteries. Adv. Sci. 5:1800080. doi: 10.1002/advs.201800080

Hahm, M., Reddy, A., Cole, D., Rivera, M., Vento, J., Nam, J., et al. (2012). Carbon nanotube-nanocup hybrid structures for high power supercapacitor applications. Nano Lett. 12, 5616-5621. doi: 10.1021/nl3027372

Han, S., Wu, D., Li, S., Zhang, F., and Feng, X. (2014). Porous graphene materials for advanced electrochemical energy storage and conversion devices. Adv. Mater. Weinheim. 26, 849-864. doi: 10.1002/adma.201303115

Han, Y., Hu, J., Yin, C., Zhang, Y., and Xie, J. (2016). Iron-based fluorides of tetragonal tungsten bronze structure as potential cathodes for $\mathrm{Na}$-ion batteries. J. Mater. Chem. A. 4, 7382-7389. doi: 10.1039/C6TA02061E

Hao, L., Li, X., and Zhi, L. (2013). Carbonaceous electrode materials for supercapacitors. Adv. Mater. Weinheim. 25, 3899-3904. doi: 10.1002/ adma.201301204

Hou, H., Banks, C. E., Jing, M., Zhang, Y., and Ji, X. (2015). Carbon quantum dots and their derivative $3 \mathrm{D}$ porous carbon frameworks for Sodium-ion batteries with ultralong cycle life. Adv. Mater. Weinheim. 27, 7861-7866. doi: 10.1002/adma.201503816

Hou, H., Shao, L., Zhang, Y., Zou, G., Chen, J., and Ji, X. (2017), Large-area carbon nanosheets doped with phosphorus: a high-performance anode material for sodium-ion batteries. Adv. Sci. 4:1600243. doi: 10.1002/advs.201600243

Jin, H., Feng, X., Li, J., Li, M., Xia, Y., Yuan, Y., et al. (2018a). Heteroatomdoped porous carbon materials with unprecedented high volumetric capacitive performance. Angew. Chem. Int. Ed. 58, 2397-2401. doi: 10.1002/anie.201813686

Jin, H., Li, J., Yuan, Y., Wang, J., Lu, J., and Wang, S. (2018b). Recent progress in biomass-derived electrode materials for high volumetric performance supercapacitors. Adv. Energy Mater. 8:1801007. doi: 10.1002/aenm. 201801007

Kang, D., Liu, Q., Gu, J., Su, Y., Zhang, W., and Zhang, D. (2015). "Egg-Box"-assisted fabrication of porous carbon with small mesopores for high-rate electric double layer capacitors. ACS Nano. 9, 11225-11233. doi: 10.1021/acsnano.5b04821 
Kong, D., Gao, Y., Xiao, Z., Xu, X., Li, X., and Zhi, L. (2019). Rational design of carbon-rich materials for energy storage and conversion. Adv. Mater. Weinheim. 31:1804973. doi: 10.1002/adma.2018 04973

Li, J., Wei, H., Peng, Y., Geng, L., Zhu, L., Cao, X., et al. (2019a). A multifunctional self-healing $\mathrm{G}-\mathrm{PyB} / \mathrm{KCl}$ hydrogel: smart conductive, rapid room-temperature phase-selective gelation, and ultrasensitive detection of alpha-fetoprotein. Chem. Commun. 55, 7922-7925. doi: 10.1039/C9CC 02770J

Li, J., Yun, X., Hu, Z., Xi, L., Li, N., Tang, H., et al. (2019b). Three-dimensional nitrogen and phosphorus co-doped carbon quantum dots/reduced graphene oxide composite aerogels with a hierarchical porous structure as superior electrode materials for supercapacitors. J. Mater. Chem. A. 7, 26311-26325. doi: 10.1039/C9TA08151H

Li, L., Wu, Z., Sun, H., Chen, D., and Gao, J. (2015). A foldable lithiumsulfur battery. ACS Nano. 9, 11342-11350. doi: 10.1021/acsnano. $5 b 05068$

Li, W., Zhang, F., Dou, Y., Wu, Z., Liu, H., Qian, X., et al. (2011). A self-template strategy for the synthesis of mesoporous carbon nanofibers as advanced supercapacitor electrodes. Adv. Energy Mater. 1, 382-386. doi: 10.1002/aenm.201000096

Liu, C., Zhang, Z., Chen, M., Zhao, H., Duan, F., Chen, D., et al. (2017). Pore modulation of zirconium-organic frameworks for high efficiency detection of trace proteins. Chem. Commun. 53, 3941-3944. doi: 10.1039/C7CC00029D

Liu, D., Ni, K., Ye, J., Xie, J., Zhu, Y., and Song, L. (2018). Tailoring the structure of carbon nanomaterials toward high-end energy applications. Adv. Mater. Weinheim. 30:1802104. doi: 10.1002/adma.201802104

Lv, J., Liang, T., Yang, M., Ken, S., and Hideo, M. (2017a). Performance comparison of $\mathrm{NiCo}_{2} \mathrm{O}_{4}$ and $\mathrm{NiCo}_{2} \mathrm{~S}_{4}$ formed on $\mathrm{Ni}$ foam for supercapacitor. Compos. Part B-Eng. 123, 28-33. doi: 10.1016/j.compositesb.2017.05.021

Lv, J., Yang, M., Liang, T., and Miura, H. (2017b). Facile synthesis of $\mathrm{Co}_{3} \mathrm{O}_{4} @ \mathrm{MnO}_{2}$ core-shell nanocomposites for high-performance supercapacitor. Mater. Lett. 197, 127-130. doi: 10.1016/j.matlet.2017.03.127

Lv, Y., Gan, L., Liu, M., Xiong, W., Xu, Z., Zhu, D., et al. (2012). A self-template synthesis of hierarchical porous carbon foams based on banana peel for supercapacitor electrodes. J. Power Sour. 209, 152-157. doi: 10.1016/j.jpowsour.2012.02.089

Mun, Y., Jo, C., Hyeon, T., Lee, J., Ha, K., Jun, K., et al. (2013). Simple synthesis of hierarchically structured partially graphitized carbon by emulsion/blockcopolymer co-template method for high power supercapacitors. Carbon N. Y. 64, 391-402. doi: 10.1016/j.carbon.2013.07.092

Nawwar, M., Poon, R., Chen, R., Sahu, R. P., Puri, I. K., and Zhitomirsky, I. (2019). High areal capacitance of $\mathrm{Fe}_{3} \mathrm{O}_{4}$-decorated carbon nanotubes for supercapacitor electrodes. Carbon Energy 1, 124-133. doi: 10.1002/cey2.6

$\mathrm{Ni}$, J., and Li, Y. (2016). Carbon nanomaterials in different dimensions for electrochemical energy storage. Adv. Energy Mater. 6:1600278. doi: 10.1002/aenm.201600278

Péan, C., Merlet, C., Rotenberg, B., Madden, P., Taberna, P., Daffos, B., et al. (2014). On the dynamics of charging in nanoporous carbon-based supercapacitors. ACS Nano. 8, 1576-1583. doi: 10.1021/nn4058243

Presser, V., Zhang, L., Niu, J., McDonough, J., Perez, C., Fong, H., et al. (2011). Flexible nano-felts of carbide-derived carbon with ultra-high power handling capability. Adv. Energy Mater. 1, 423-430. doi: 10.1002/aenm.201100047

Ra, E. J., Raymundo-Piñero, E., Lee, Y. H., and Béguin, F. (2009). High power supercapacitors using polyacrylonitrile-based carbon nanofiber paper. Carbon N. Y. 47, 2984-2992. doi: 10.1016/j.carbon.2009.06.051

Raymundo-Piñero, E., Cadek, M., Wachtler, M., and Béguin, F. (2011). Carbon nanotubes as nanotexturing agents for high power supercapacitors based on seaweed carbons. ChemSusChem. 4, 943-949. doi: 10.1002/cssc.2010 00376

Shi, D., Zheng, R., Liu, C., Chen, D., Zhao, J., and Du, M. (2019). Dual functionalized mixed keggin- and lindqvist-type $\mathrm{Cu}_{24}$-based POM@MOF for visible-light-driven $\mathrm{H}_{2}$ and $\mathrm{O}_{2}$ Evolution. Inorg. Chem. 58, 7229-7235. doi: 10.1021 /acs.inorgchem. 9 b00206

Shi, D., Zheng, R., Sun, M., Cao, X., Sun, C., Cui, C., et al. (2017). Semi-conductive copper (I)-organic frameworks for efficient light-driven hydrogen generation without additional photosensitizers and cocatalysts. Angew. Chem. Int. Ed. 56, 14637-14641. doi: 10.1002/anie.201709869
Sun, L., Tian, C., Li, M., Meng, X., Wang, L., Wang, R., et al. (2013). From coconut shell to porous graphene-like nanosheets for high-power supercapacitors. $J$. Mater. Chem. A. 1, 6462-6470. doi: 10.1039/c3ta10897j

Wang, F., Wu, X., Yuan, X., Liu, Z., Zhang, Y., Fu, L., et al. (2017). Latest advances in supercapacitors: from new electrode materials to novel device designs. Chem. Soc. Rev. 46, 6816-6854. doi: 10.1039/c7cs0 0205J

Wang, H., and Cui, Y. (2019). Nanodiamonds for energy. Carbon Energy. 1, 13-18. doi: $10.1002 /$ cey2.9

Wang, L., Dubin, M., Hwang, J., Shao, Y., Marsh, K., McVerry, B., et al. (2015). Flash converted graphene for ultra-high power supercapacitors Adv. Energy Mater. 5:1500786. doi: 10.1002/aenm.201500786

Wang, Y., and Xia, Y. (2013). Recent progress in supercapacitors: From materials design to system construction. Adv. Mater. Weinheim. 25, 5336-5342. doi: 10.1002/adma.201301932

Wen, L., Li, F., and Cheng, H. (2016). Carbon nanotubes and graphene for flexible electrochemical energy storage: from materials to devices. Adv. Mater. Weinheim. 28, 4306-4337. doi: 10.1002/adma.201504225

Wu, T., Zhang, C., Zou, G., Hu, J., Zhu, L., Cao, X., et al. (2019). The bond evolution mechanism of covalent sulfurized carbon during electrochemical sodium storage process. Sci. Chi. Mater. 62, 1127-1138. doi: 10.1007/s40843-019-9418-8

Xie, K., Qin, X., Wang, X., Wang, Y., Tao, H., Wu, Q., et al. (2012). Carbon nanocages as supercapacitor electrode materials. Adv. Mater. Weinheim. 24, 347-352. doi: 10.1002/adma.201103872

Xu, B., Duan, H., Liu, H., Wang, C., and Zhong, S. (2017). Stabilization of garnet/liquid electrolyte interface using superbase additives for hybrid $\mathrm{Li}$ batteries. ACS Appl. Mater. Inter. 9, 21077-21082. doi: 10.1021/acsami.7b05599

Xu, M., Fei, L., Lu, W., Chen, Z., Li, T., Liu, Y., et al. (2017). Engineering hetero-epitaxial nanostructures with aligned Li-ion channels in Li-rich layered oxides for high-performance cathode application. Nano Energy 35, 271-280. doi: 10.1016/j.nanoen.2017.03.051

Xu, Y., Lin, Z., Zhong, X., Huang, X., Weiss, N., Huang, Y., et al. (2014). Holey graphene frameworks for highly efficient capacitive energy storage. Nat. Commun. 5:4554. doi: 10.1038/ncomms5554

Yang, L., Hong, W., Tian, Y., Zou, G., Hou, H., Sun, W., et al. (2019). Heteroatom-doped carbon inlaid with $\mathrm{Sb}_{2} \mathrm{X}_{3}(\mathrm{X}=\mathrm{S}$, Se) nanodots for high-performance potassium-ion batteries. Chem. Eng. J. 385:123838. doi: $10.1016 /$ j.cej.2019.123838

Yang, M., and Zhou, Z. (2017). Recent breakthroughs in supercapacitors boosted by nitrogen-rich porous carbon materials. Adv. Sci. 4:1600408 doi: 10.1002/advs.201600408

Ye, J., Simon, P., and Zhu, Y. (2019). Designing ionic channels in novel carbons for electrochemical energy storage. Nat. Sci. Rev. 13, 2095-5138. doi: 10.1093/nsr/nwz140

Yin, Y., Yu, Z., Ma, Z., Zhang, T., Lu, Y., Ma, T., et al. (2018). Bio-inspired lowtortuosity carbon host for high-performance lithium-metal anode. Nat.Sci.Rev. 6, 247-256. doi: 10.1093/nsr/nwy148

Yu, P., Zhang, Z., Zheng, L., Teng, F., Hu, L., and Fang, X. (2016). A novel sustainable flour derived hierarchical nitrogen-doped porous carbon/polyaniline electrode for advanced asymmetric supercapacitors. Adv. Energy Mater. 6:1601111. doi: 10.1002/aenm.201601111

Yuan, Y., and Lu, J. (2019). Demanding energy from carbon. Carbon Energy. 1, 8-12. doi: $10.1002 /$ cey2.12

Zhang, F., Liu, T., Li, M., Yu, M., Luo, Y., Tong, Y., et al. (2017). Multiscale pore network boosts capacitance of carbon electrodes for ultrafast charging. Nano Lett. 17, 3097-3104. doi: 10.1021/acs.nanolett.7b00533

Zheng, S., Ju, H., and Lu, X. (2015). A high-performance supercapacitor based on $\mathrm{KOH}$ activated 1D C 70 Microstructures. Adv. Energy Mater. 5:1500871. doi: 10.1002/aenm.201500871

Zhou, H., Cao, Y., Ma, Z., and Li, S. (2018). Facile synthesis of nickel-doped $\mathrm{Co}_{9} \mathrm{~S}_{8}$ hollow nanoparticles with large surface-controlled pseudocapacitive and fast sodium storage. Nanotechnology 29, 195-201. doi: 10.1088/1361-6528/ aab120

Zhou, H., and Hu, J. (2017). Facile synthesis of multi-walled carbon nanotubes $/ \mathrm{Co}_{9} \mathrm{~S}_{8}$ composites with enhanced performances for sodiumion battery. Mater. Lett. 175, 26-30. doi: 10.1016/j.matlet.2017. 02.004 
Zhu, H., Wang, X., Yang, F., and Yang, X. (2011). Promising carbons for supercapacitors derived from fungi. Adv. Mater. Weinheim. 23, 2745-2748. doi: 10.1002/adma.201100901

Zhu, J., Childress, A., Karakaya, M., Dandeliya, S., Srivastava, A., Lin, Y., et al. (2016a). Defect-engineered graphene for high-energy- and highpower-density supercapacitor devices. Adv. Mater. Weinheim. 28, 7185-7192. doi: 10.1002/adma.201602028

Zhu, J., Xu, Y., Zhang, Y., Feng, T., Wang, J., Mao, S., et al. (2016b). Porous and high electronic conductivity nitrogen-doped nano-sheet carbon derived from polypyrrole for high-power supercapacitors. Carbon N. Y. 107, 638-645. doi: 10.1016/j.carbon.2016. 06.063

Zhu, Y., Ji, H., Cheng, H., and Ruoff, R. (2017). Mass production and industrial applications of graphene materials. Nat. Sci. Rev. 5, 90-101. doi: 10.1093/nsr/nwx055

Zhu, Y., Li, J., Yun, X., Zhao, G., Ge, P., Zou, G., et al. (2020). Graphitic carbon quantum dots modified nickel cobalt sulfide as cathode materials for alkaline aqueous batteries. Nano-Micro Lett. 12, 1-18. doi: 10.1007/s40820-0190355-0

Zou, G., Hou, H., Foster, W., Banks, C. E., Guo, T., Jiang, Y., et al. (2018). Advanced hierarchical vesicular carbon Co-doped with S, P, N for high-rate sodium storage. Adv. Sci. 5:1800241. doi: 10.1002/advs.201800241

Conflict of Interest: The authors declare that the research was conducted in the absence of any commercial or financial relationships that could be construed as a potential conflict of interest.

Copyright (c) 2020 Cai, Zou, Deng, Wang, Zou, Hou and Ji. This is an open-access article distributed under the terms of the Creative Commons Attribution License (CC BY). The use, distribution or reproduction in other forums is permitted, provided the original author(s) and the copyright owner(s) are credited and that the original publication in this journal is cited, in accordance with accepted academic practice. No use, distribution or reproduction is permitted which does not comply with these terms. 